\title{
Cost-effectiveness analysis of malaria rapid diagnostic tests: a systematic review
}

\author{
Xiao-Xiao Ling', Jia-Jie Jin', Guo-Ding Zhu ${ }^{2,3,4}$, Wei-Ming Wang², Yuan-Yuan Cao², Meng-Meng Yang², \\ Hua-Yun Zhou', Jun Cao ${ }^{2,3,4^{*}}$ and Jia-Yan Huang ${ }^{1 *}$ id
}

\begin{abstract}
Background: Rapid diagnostic tests (RDT) can effectively manage malaria cases and reduce excess costs brought by misdiagnosis. However, few studies have evaluated the economic value of this technology. The purpose of this study is to systematically review the economic value of RDT in malaria diagnosis.

Main text: A detailed search strategy was developed to identify published economic evaluations that provide evidence regarding the cost-effectiveness of malaria RDT. Electronic databases including MEDLINE, EMBASE, Biosis Previews, Web of Science and Cochrane Library were searched from Jan 2007 to July 2018. Two researchers screened studies independently based on pre-specified inclusion and exclusion criteria. The Consolidated Health Economic Evaluation Reporting Standards (CHEERS) checklist was applied to evaluate the quality of the studies. Then cost and effectiveness data were extracted and summarized in a narrative way. Fifteen economic evaluations of RDT compared to other diagnostic methods were identified. The overall quality of studies varied greatly but most of them were scored to be of high or moderate quality. Ten of the fifteen studies reported that RDT was likely to be a cost-effective approach compared to its comparisons, but the results could be influenced by the alternatives, study perspectives, malaria prevalence, and the types of RDT.
\end{abstract}

Conclusions: Based on available evidence, RDT had the potential to be more cost-effective than either microscopy or presumptive diagnosis. Further research is also required to draw a more robust conclusion.

Keywords: Malaria, Rapid diagnostic test, Microscopy, Presumptive diagnosis, Cost-effectiveness analysis

\section{Background}

For years, the quality-assured malaria diagnosis has been emphasized to effectively control malaria cases and reduce excess costs due to misdiagnosis [1-4]. With the development of malaria control interventions and the shift towards malaria elimination globally, many countries face the new challenge of increasing imported cases due to the growing human migration and travel to the malaria-endemic region [5-7]. Failing to identify and track malaria cases promptly may hinder the realization of disease elimination and impose a substantial financial burden given the higher treatment costs and public

\footnotetext{
* Correspondence: caojuncn@hotmail.com; jiayanhuang@fudan.edu.cn ${ }^{2}$ National Health Commission Key Laboratory of Parasitic Disease Control and Prevention, Jiangsu Provincial Key Laboratory on Parasite and Vector Control Technology, Jiangsu Institute of Parasitic Diseases, Wuxi 214064, China ${ }^{1}$ School of Public Health, Fudan University, Key Laboratory of Health Technology Assessment, National Health Commission, Shanghai 200032, China

Full list of author information is available at the end of the article
}

spending. This arouses a wide concern among policymakers regarding how available malaria diagnostic methods can achieve the goal of the disease control and elimination, and whether existing packages of feasible interventions can be sustainably affordable [8].

So far, a remarkable improvement in the malaria diagnosis has been observed. Several malaria diagnostic methods are available for policymakers to choose: presumptive diagnosis, blood smear microscopy, polymerase chain reaction (PCR), and rapid diagnostic test (RDT) [9]. The presumptive diagnosis of malaria is a conventional approach that diagnoses patients based on their symptoms and clinical signs and it is still widely adopted [10]. However, it has been acknowledged that the method may add to the difficulty in effectively and accurately diagnosing the disease, and lead to a high proportion of misdiagnosis and overuse of drugs. Blood smear microscopy has advantages in both accuracy and ability to quantify parasites if it could be used properly

(c) The Author(s). 2019 Open Access This article is distributed under the terms of the Creative Commons Attribution 4.0 International License (http://creativecommons.org/licenses/by/4.0/), which permits unrestricted use, distribution, and 
[11]. But it has high requirements for technicians' skills and experience, which is difficult to guarantee especially in low transmission sites [12]. It also takes a longer time to operate, far from current expectations of an accurate and timely technique for routine malaria detection [13]. PCR is appealing for its high diagnostic accuracy. On the other side, it is most costly and has high requirements on devices, materials, and technicians, making it inappropriate for countries with limited resources [14-16].

Rapid diagnostic test (RDT) is a quick diagnostic approach to detect malaria among malaria-suspected patients and rule out malaria among individuals without malaria. It has been found that the test is highly sensitive and specific [17]. Meanwhile, RDT is easy to perform, and the results can be read in $15-30 \mathrm{~min}$. These make it suitable for community-level health facilitates in rural areas and other endemic situations where equipment and professional microscopists are not accessible.

Given the rapid development of malaria diagnosis, enhanced case identification is operationally feasible but now the question of concern for countries embarking on malaria control and elimination is how to allocate limited resources to strengthen their current surveillance system, maintain their success and avoid the risk of reintroduction of malaria particularly when the cost is largely unknown [18]. Economic evaluations can provide evidence for policymakers to identify the diagnostic test that is cost-effective and can be sustainably applied. Few studies have been carried out to evaluate the economic value of RDT, although economic evidence is necessary, and no systematic review has been performed. This study focuses on both the costs and effects of RDT and systematically evaluates whether using RDT can be costeffective compared with other malaria diagnostic methods based on available evidence.

\section{Methods}

\section{Selection criteria}

To assess the cost-effectiveness (cost-utility or costbenefit), we only considered full economic evaluations that compared RDT with other common malaria diagnostic tools. A full economic evaluation should consist of two parts, i.e. costs and effects, and provide resource use, estimates of inputs and consequences for intervention. Studies were excluded if they did not use microscopy or PCR as the reference for malaria diagnosis.

\section{Search strategy}

The search was performed initially in March 2017 and updated in July 2018 in the following databases: Cochrane Library, MEDLINE, EMBASE, Web of Science and Biosis Previews. We determined our search strategy with reference to previous relevant studies and systematic reviews. It was segmented into three components: malaria, malaria diagnosis techniques, and economic evaluations. To maintain the search comprehensiveness, the search was restricted to articles with the following terms in their titles, abstracts and keywords: "malaria", and "RDT", or "rapid diagnosis test", and "cost-effectiveness", "cost-effectiveness analysis", "cost-benefit analysis", "cost-utility analysis", "economic evaluations", "cost(s)", or "economy". We did not set limitations on population and languages.

\section{Selection of studies}

Two reviewers independently screened the titles, abstracts, and keywords of all searched studies and excluded irrelevant studies based on selection criteria. Then duplicates were removed. Two reviewers independently read the fulltext version of each study and decided whether they should be included. Disagreements on inclusion were resolved by discussion or inviting another reviewer to judge according to the same information. Studies were named by the surname of their first author and the year of publication.

\section{Data extraction}

Two reviewers independently extracted data based on a well-designed data extraction table, summarized important information and made a descriptive analysis to draw a conclusion about the cost-effectiveness of RDT.

The following data were extracted:

(1) General information: first author, study year, country, the prevalence of malaria, source of funding, participants of the study, intervention and its comparisons, commercial name of RDT, the type of RDT.

(2) Methodological information: types of study (costeffectiveness analysis, cost-utility analysis, and costbenefit analysis), study design, study perspective, time horizon, outcome measures, discount rate, currency, price year and willingness to pay threshold.

(3) Results and conclusions: incremental costs, incremental effectiveness, incremental costeffectiveness ratio (ICER) reported, and sensitivity analysis.

\section{Quality assessment}

Strict quality assessment can reflect the methodological quality of health economics research and control risk of bias. The Consolidated Health Economic Evaluation Reporting Standards (CHEERS, http://www.equator-net work.org/wp-content/uploads/2013/04/Revised-CHEERSChecklist-Oct13.pdf), a comprehensive quality assessment tool recommended by the International Society for Pharmacoeconomics and Outcomes Research, were used to 
examine the quality of studies. The CHEERS checklist assesses the reporting quality based on items from the following aspects: "title and abstract", "introduction", "methods", "discussion" and "other". Each item would be appraised critically in accordance with the requirements of CHEERS statements. The evaluation results were shown as 'Yes', 'No' and 'Not clear' marked as 1, 0 and 0 respectively, then the scores of the 24 items were summed up to calculate the final score of each study. Scores would be divided into three levels to identify the quality of each article: high (studies that met over $75 \%$ items or scored between 19 and 24), moderate (studies that met 50-75\% items or scored between 13 and 18) and low (studies that met less than $50 \%$ items or scored 12 or lower).

\section{Analysis methods}

As meta-analysis or other quantitative synthesis methods are not recommended to combine the cost-effectiveness from different economic evaluations [19], we summarized results of each study in a narrative way and presented incremental costs, incremental effectiveness, and ICERs in structured tables if such information was available.

The narrative and tabular summary were structured based on the age of the population and the perspective of economic evaluations since participants' age might influence the economic results of the intervention [20-22], and the perspectives could determine the costs and effects that were included in the evaluations. We also recognized that there might be potential impact brought by the type of RDTs and funding sources on the economic values of diagnostic tests and took it into account in our analysis.

To facilitate the comparison across different studies, original costs reported were converted to a common currency and price year, 2019 United States dollars (USD), given the latest version of a web-based cost converter. This tool adjusts cost estimates based on purchasing power parity (PPP) and gross domestic product (GDP) deflator index and has been recommended by the guidelines of systematic review [19, 23]. ICERs were then recalculated by dividing the converted costs by the original effects. In order to graphically compare the economic value of different malaria diagnostic methods, we adopted the cost-effectiveness plane to reflect the differences in ICER, the only appropriate measure that can capture the true economic value [24], for studies that provided relevant data and took the same outcomes of effectiveness. Studies would be categorized according to the perspectives of economic evaluations. We recognized that taking narrower perspectives (e.g., a provider perspective) could impede the comparability of the results throughout healthcare systems and ignore the implication of opportunity costs brought by the introduction of new technologies $[25,26]$. Thus we selected results from studies taken the societal perspective and the same outcome measures and plotted them on the same costeffectiveness plane.

\section{Results}

One thousand seven hundred forty studies were identified from electronic databases. After screening the titles, abstracts, and keywords, 1595 studies were removed based on inclusion and exclusion criteria, 85 studies were removed due to the duplicates and 60 full-text potentially eligible articles were retrieved for the consideration. Finally, 15 studies were included in the analysis [27-41]. The flow diagram of our study selection is shown in Fig. 1.

\section{General characteristics of studies}

We included fifteen studies that compared the economic value of RDT with other malaria diagnostic methods. Fourteen studies were full health economic evaluations that made a comparison in terms of costs and effectiveness between RDT and its comparators. All of them were cost-effectiveness analyses, nine of which used decision tree models. Besides, one study, although did not say that it was a cost-effectiveness analysis, assessed both the costs and the specificity of RDT, thus we also considered it as full economic evaluations and included it [28] (Table 1).

Most of the studies were conducted in Africa, except three: one in Afghanistan [29], and two in Brazil [36, 37]. The Africa-based studies were all performed in SubSaharan Africa (Ethiopia [32], Congo [35], Ghana [38, 40], Kenya [28], Nigeria [41], Senegal [34], Tanzania [33], Uganda [27, 30, 31]). One study targeted at all endemic countries in Sub-Saharan Africa using a simulated cohort with fever in the rural areas [39].

Eleven studies focused on suspected malaria and fever patients. Among the other four studies, two targeted at children [38, 40], one focused on the application of RDT in school students [28], and one assessed the effectiveness of RDT among healthy pregnant women [35].

\section{Quality assessment}

According to the CHEERS checklist, huge gaps existed in the quality of evidence reported. Scores ranged from 7 to 23 . Two studies provided a high quality of evidence with the highest score of $23[29,40]$, five had evidence of moderate quality [27, 30, 35, 36, 38], and eight had low quality with the lowest score of $7[28,31-34,37,39$, 41]. The overall quality of all studies included could be seen in Fig. 2 and Additional file 1.

\section{The cost-effectiveness of RDT}

The economic value of RDT was assessed in the fifteen economic evaluations and summarized in Table 2. Three 


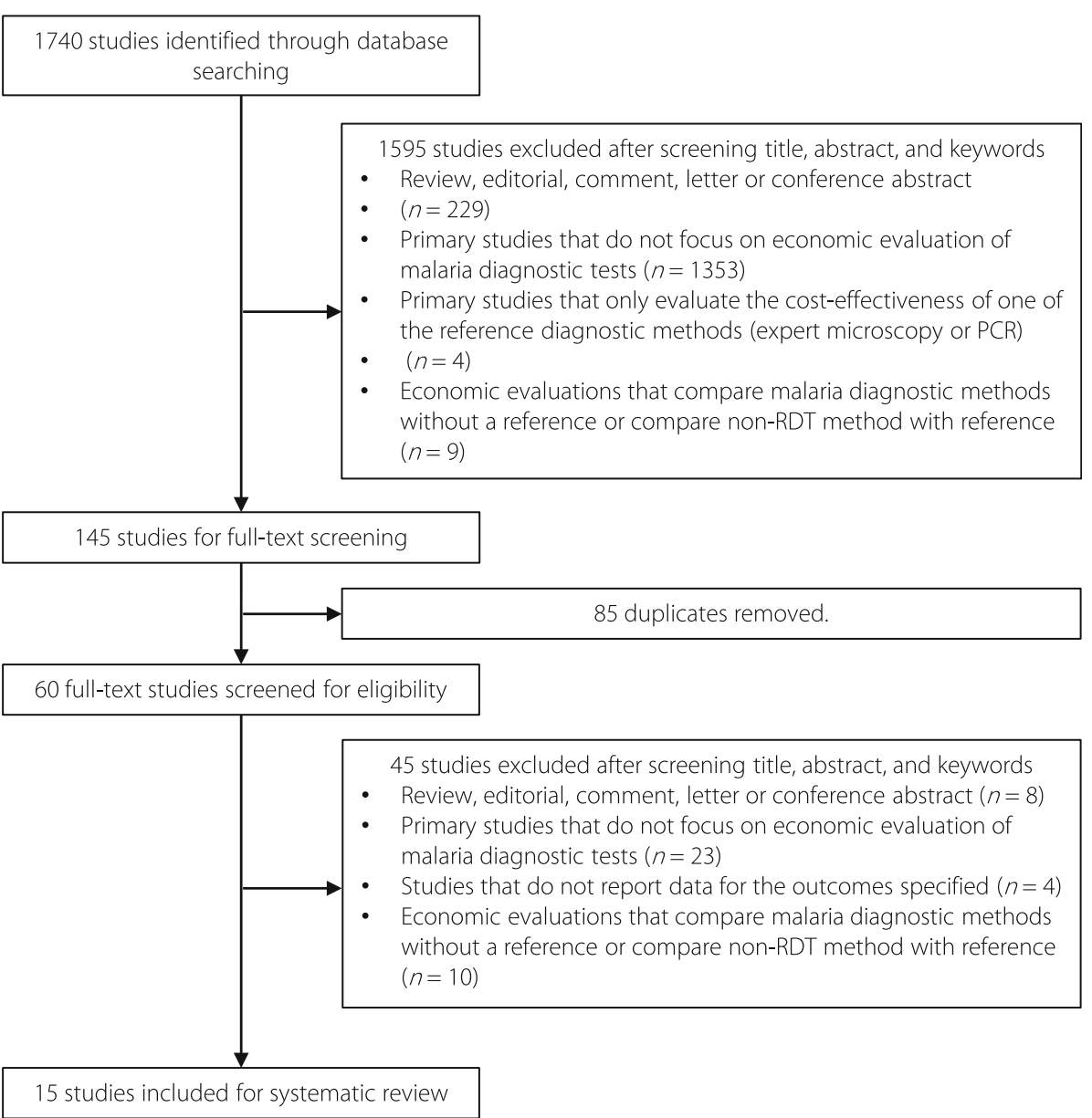

Fig. 1 Study flow diagram. Flowchart showing inclusion and exclusion process of study identification. RDT: Rapid diagnostic test

malaria diagnostic techniques were reported and compared in all papers: RDT, microscopy, and presumptive diagnosis, and the majority took microscopy and/or presumptive diagnosis method as the comparison for RDT.

\section{RDT vs microscopy}

Microscopy is a conventional diagnostic method to detect malaria infection. Six out of fifteen studies found that introducing RDT to substitute microscopy was likely to be cost-effective $[27,29,35,37,39,41]$. Four of them made that conclusion as RDT could lead to either lower costs and improved outcomes, or a cost-saving when compared to microscopy [29, 35, 37, 41]. A costeffectiveness analysis based on decision tree compared RDT and microscopy to presumptive diagnosis simultaneously [27]. It found that overall, RDT had lower positive ICER than microscopy and was most cost-effective in both high and low transmission settings. A decisionanalytical study presented evidence of the costeffectiveness of RDT compared to both microscopy and presumptive diagnosis [39]. With a threshold of USD
150 for the incremental cost per addition averted disability-adjusted life years (DALYs), RDT was highly likely to be cost-effective.

\section{RDT vs presumptive diagnosis}

The cost-effectiveness of RDT in comparison to the presumptive diagnostic method was reported in ten studies, and all of them used presumptive diagnosis as a base case with RDT as the intervention to compare [27, 29-32, 34, 38-41]. Eight studies provided supportive evidence that RDT was highly likely to be cost-effective: three studies observed that the use of RDT could be less costly while more effective [27, 32, 41], three studies found that RDT could result in an increase in both costs and effectiveness but it had the potential to be cost-effective at a low willingness to pay (WTP) threshold [29, 30, 40], another study observed a low ICER of RDT but admitted that whether RDT could be cost-effective would depend on how much decision-makers would be willing to pay [31], and a decision-based analysis showed that RDT was $85 \%$ certain to be cost-effective at all prevalence level below 65\% [39]. 


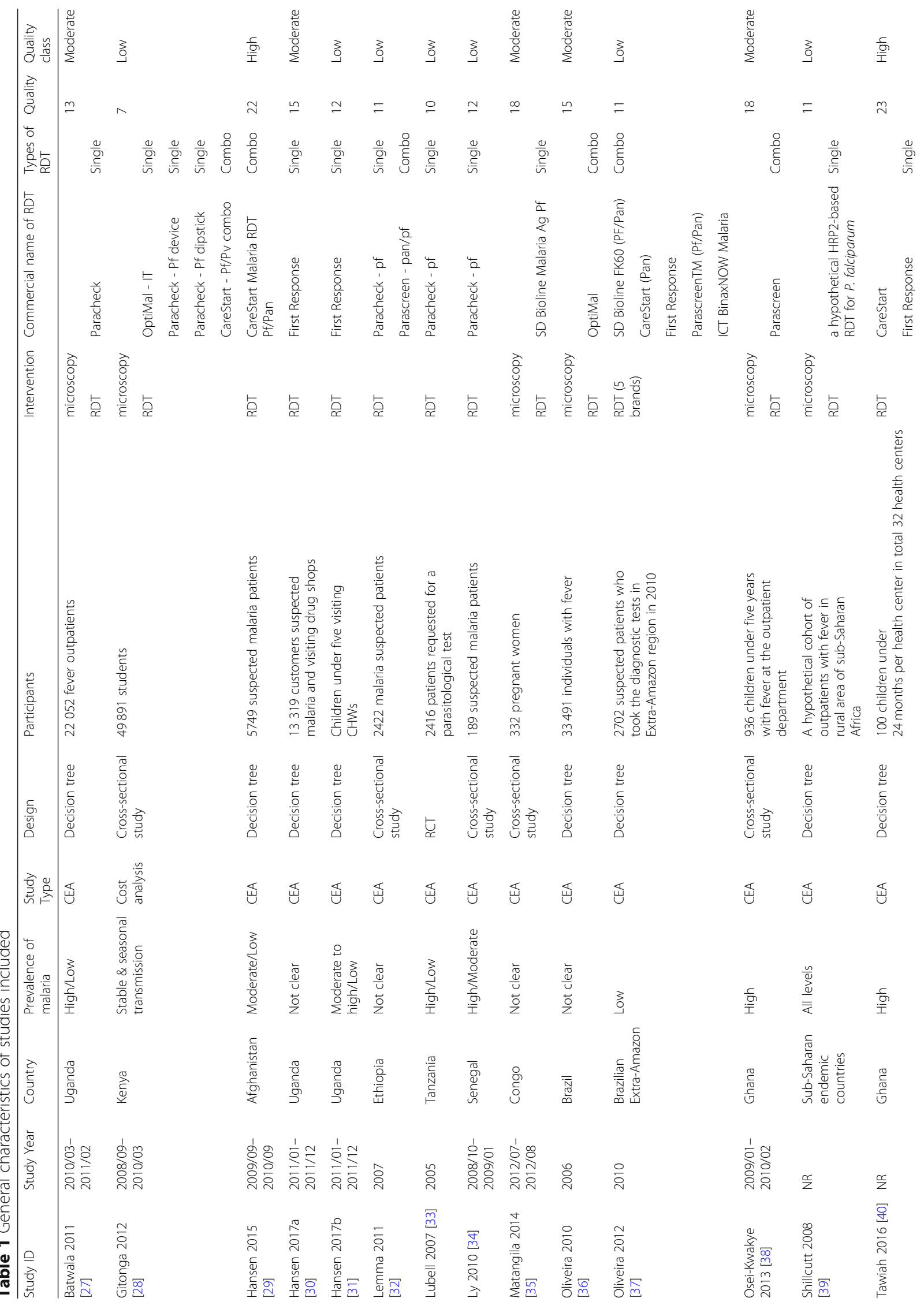




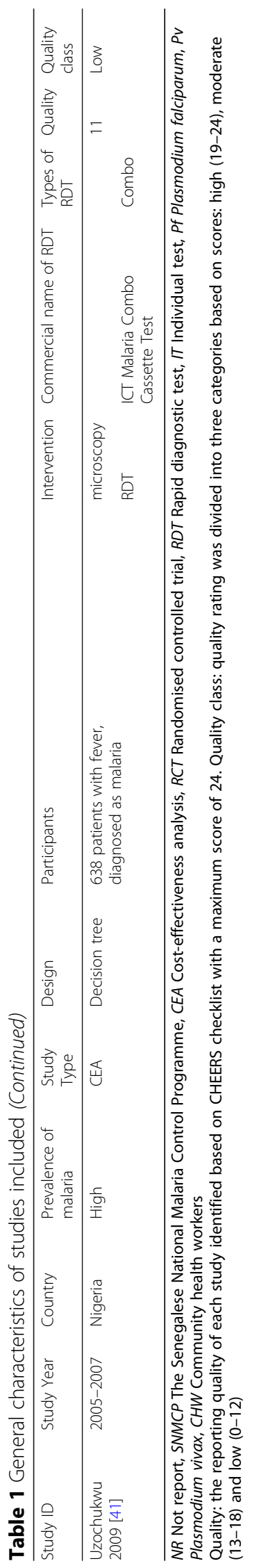




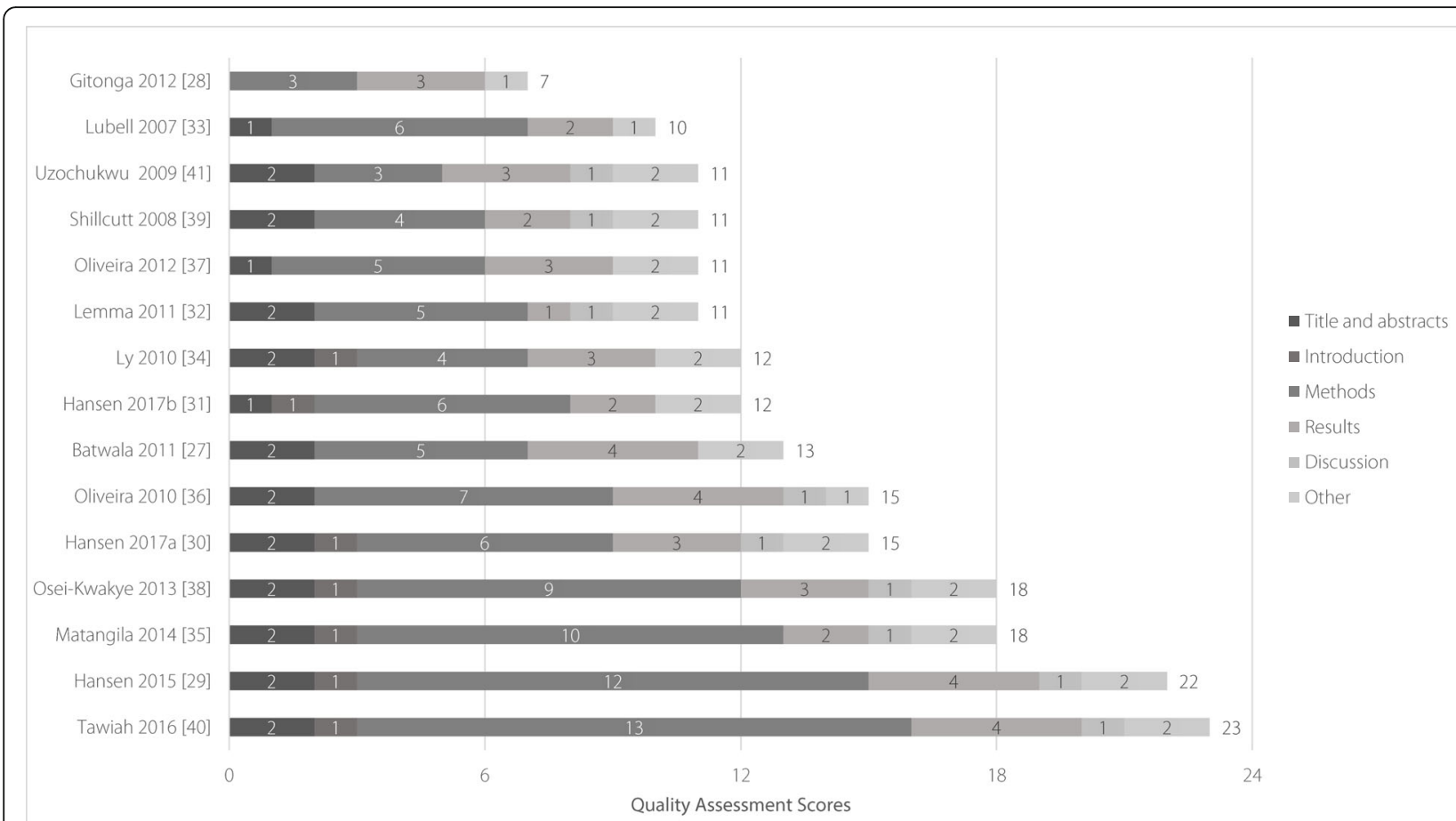

Fig. 2 Quality assessment results of studies includedThe quality of each included study was assessed based on the 24-item CHEERS checklist with a maximum score of 24 if the study could meet quality criteria for all the items.

\section{Perspectives}

Studies took a wide range of study perspectives which determined the scope of costs and effects within the evaluations: five studies were conducted from the societal perspective, four adopted a perspective of the health sector, one study did not report its perspective and the rest were undertaken under narrower perspectives such as provider or patient. There was a high level of heterogeneity among the selection of outcome measures among studies with narrow perspectives while the five studies under a societal perspective adopted either the number or the proportion of appropriately treated patients as the outcome, which can be considered as the same measure of effectiveness. We thus would take "the number of appropriately treated per 1000 suspected cases" as the main outcome and recalculate the results based on the available data.

The comparison of the economic value of RDT between five studies taking a societal perspective was plotted in Fig. 3. Compared with other diagnostic techniques, the incremental effects of RDT were always positive, i.e., using RDT could contribute to an increase in the number of appropriately treated patients, but its impact on additional societal costs was not clear and could largely depend on the comparator selected. The introduction of RDT to replace presumptive diagnosis resulted in an increase in costs $[27,29-31,40]$, but that increase was relatively small in most of the studies. There were two studies that provided evidence for the comparison between RDT and microscopy from a societal perspective, they observed a cost-saving effect when RDT was introduced [27, 29]. Overall, given a small number of studies, it could be found that RDT had the potential to be cost-effective particularly compared to microscopy under a societal perspective and whether RDT could be a dominant strategy would largely depend on the threshold of policymakers.

Similar trends could be observed when economic evaluations were undertaken under a health sector perspective. Most of them concluded that RDT was cost-effective when it was compared to microscopy and likely to be costeffective when compared with the presumptive diagnosis. RDT can keep its advantages over microscopy with lower costs and more patients appropriately treated [29, 31, 40]. It can also largely improve the clinical performance but resulted in a slight increase in the health sector costs if it was used to replace presumptive diagnosis [29-31, 40]. Further details of cost-effectiveness in studies included could be seen in Additional files 2 and 3.

\section{Prevalence}

Changes to the malaria prevalence tended to have an impact on the costs and effects of diagnostic methods. Thirteen studies recognized its potential influence on the cost-effectiveness of RDT compared to other methods but only eight of them formally investigated the uncertainty brought by malaria prevalence [27, 30, 31, 33, 37, 39-41]. 


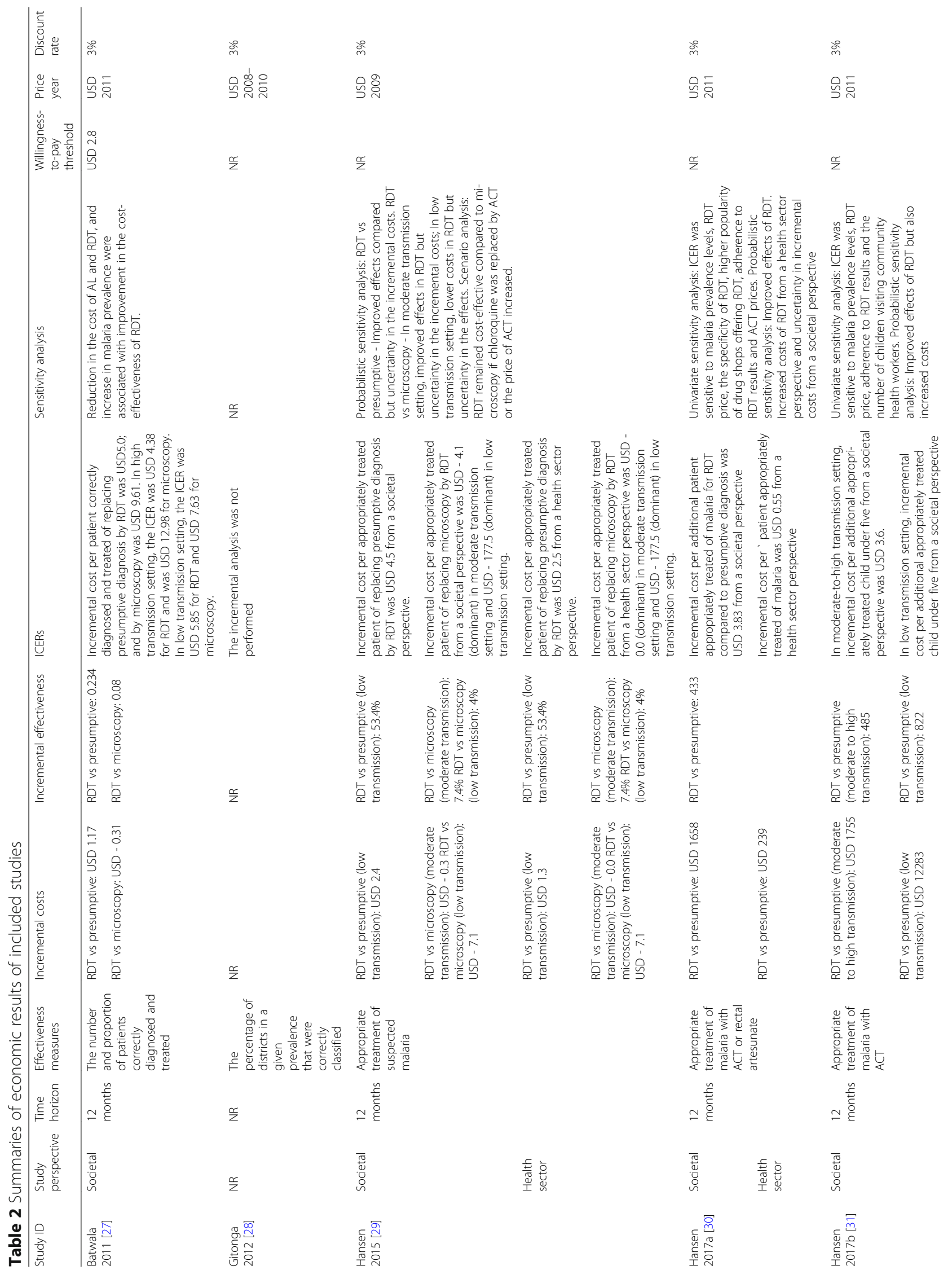




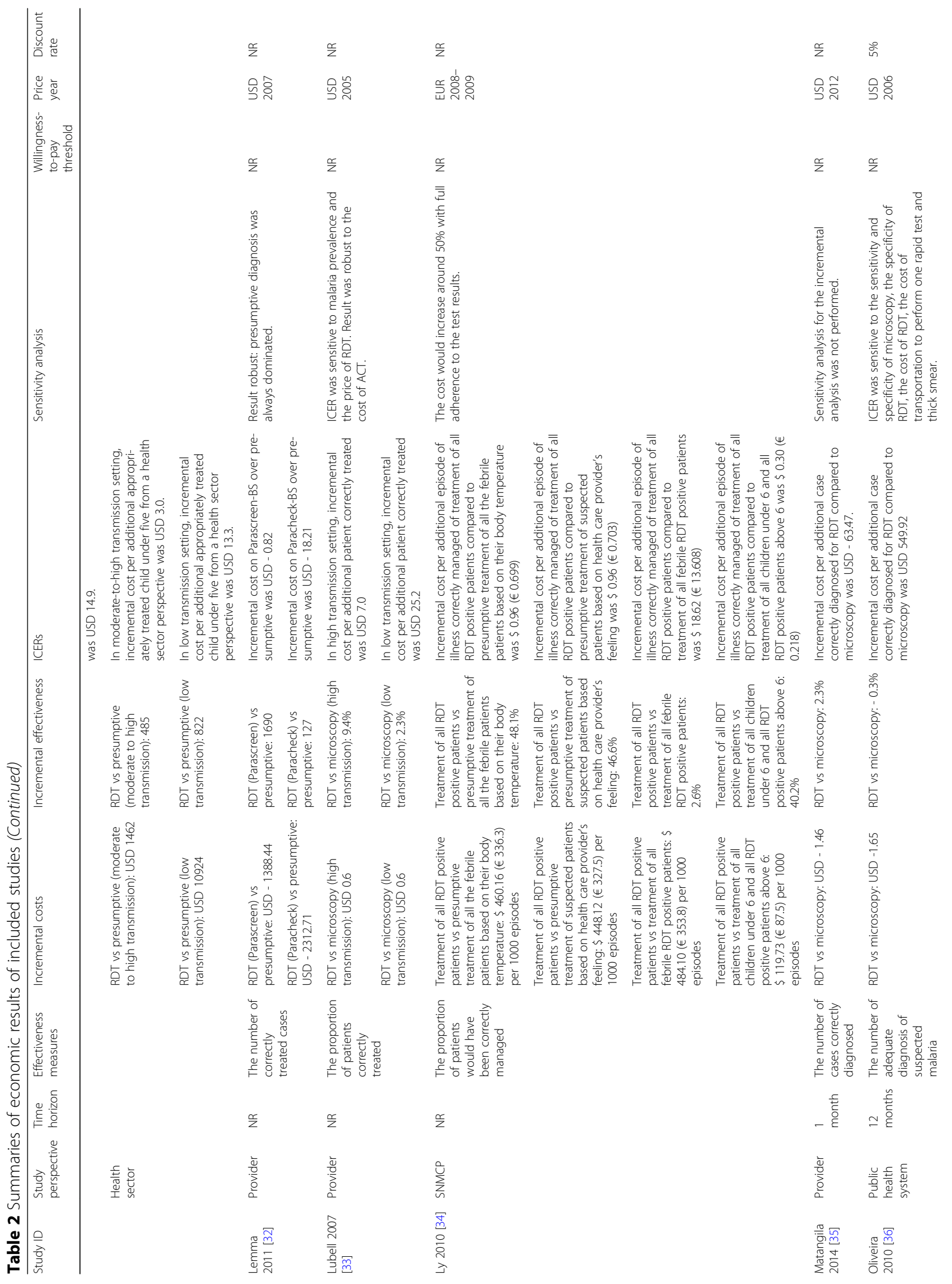




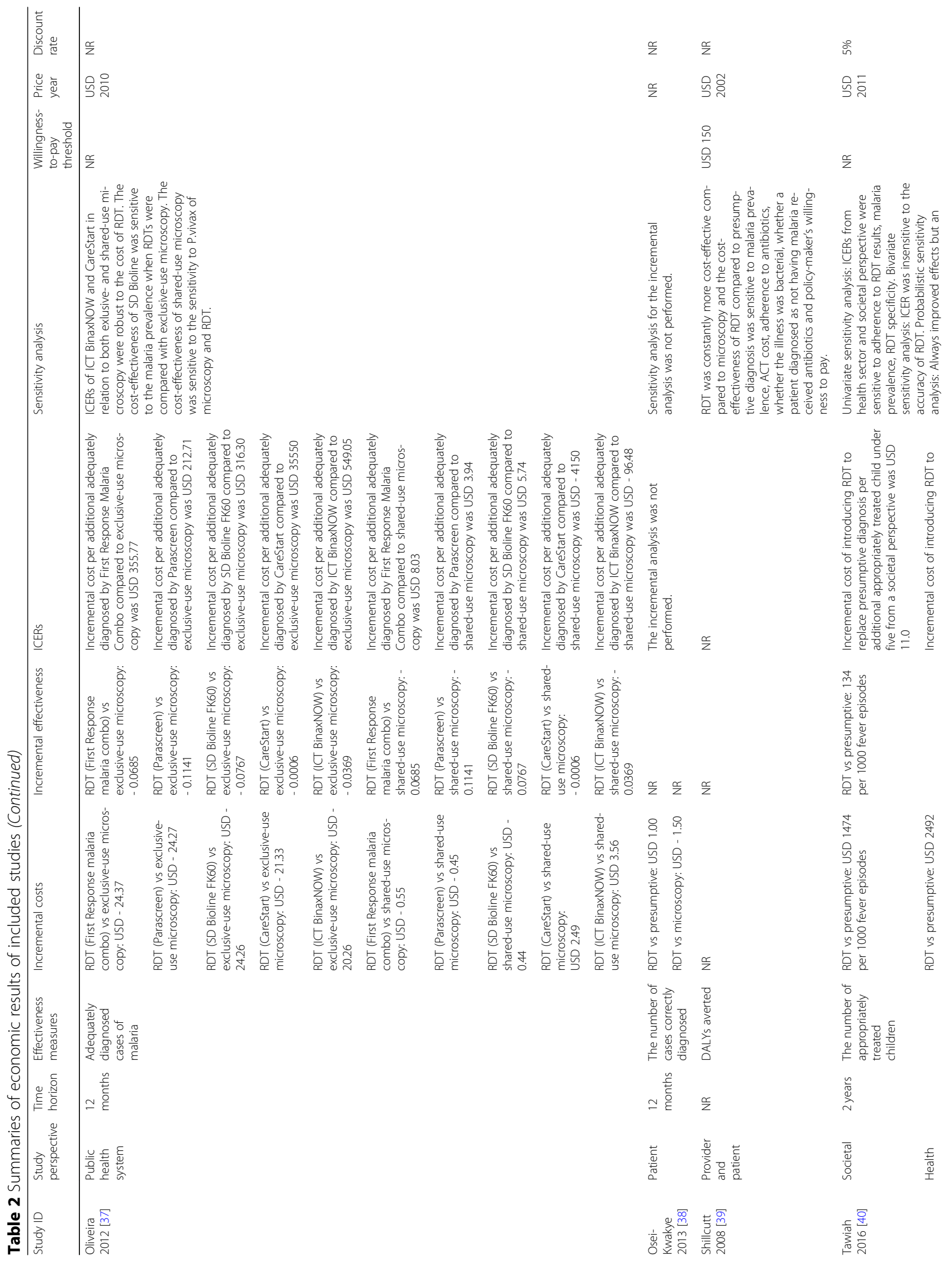




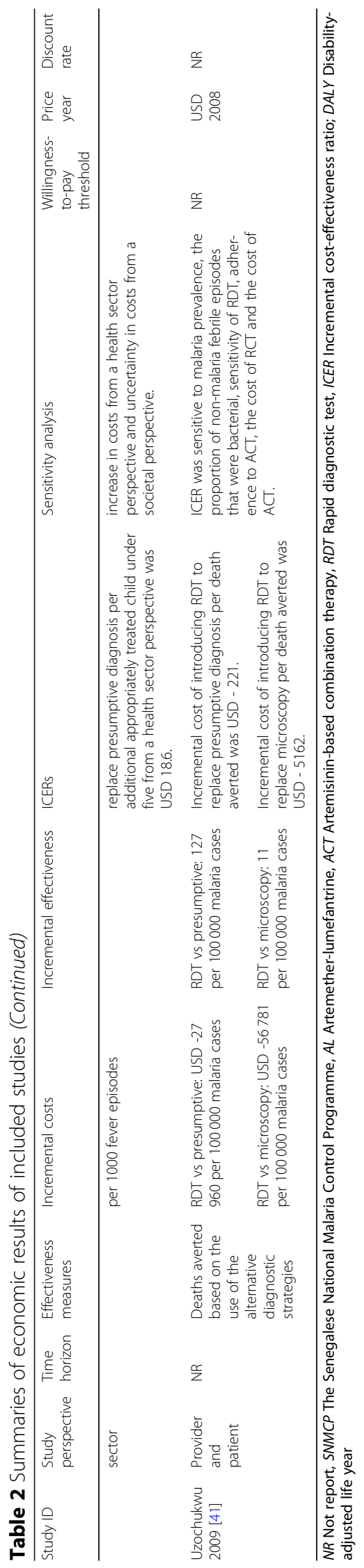




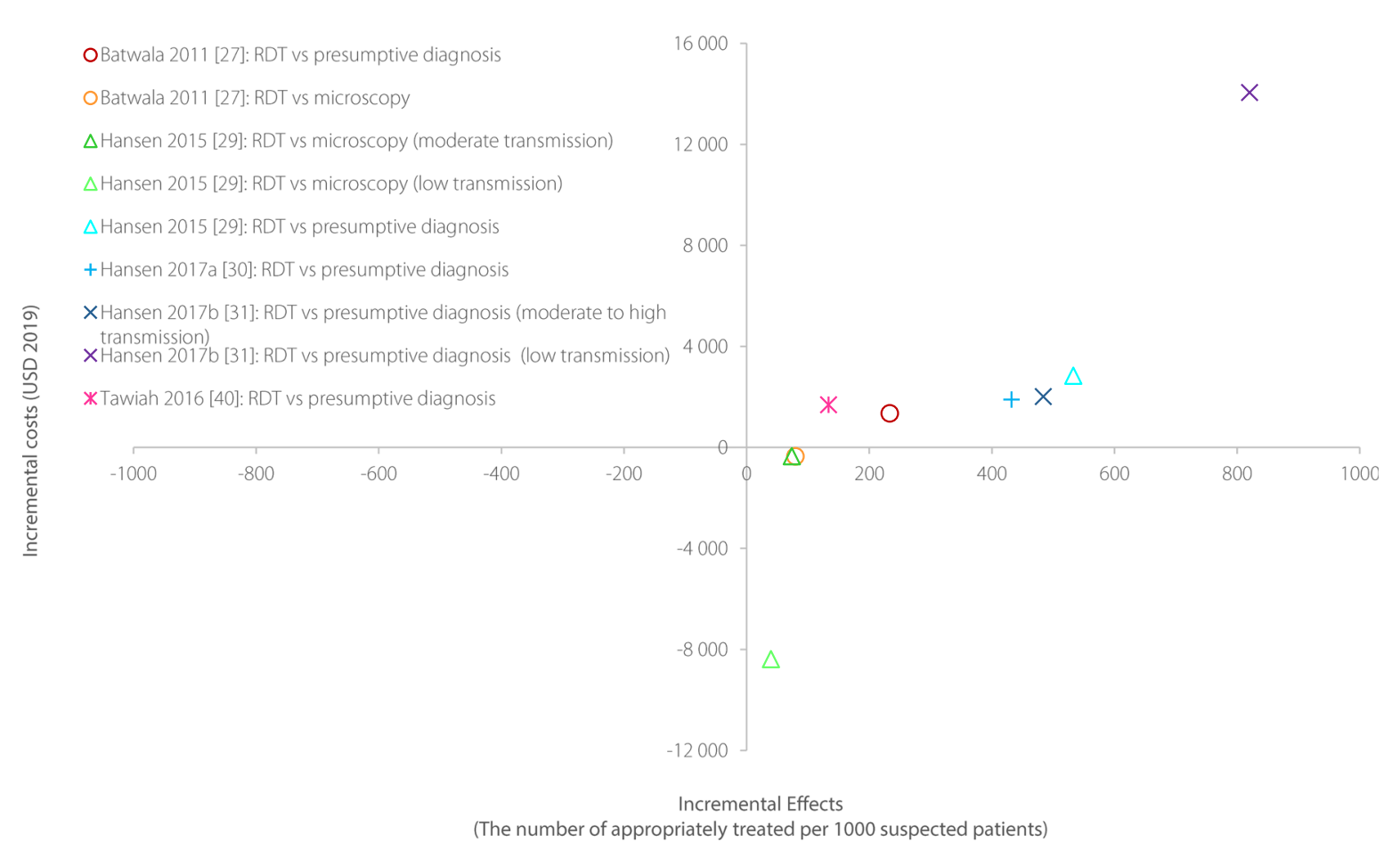

Fig. 3 Incremental cost-effectiveness ratio of studies included (societal perspective)Each point represents differences in the costs and effectiveness between RDT and its alternatives from included studies under a societal perspective.RDT: Rapid diagnostic test.

The introduction of RDT to replace microscopy was found to be a dominant strategy regardless of the prevalence levels $[27,37,39,41]$, but the ICER could be lower with an increase in prevalence [33]. The cost-effectiveness of RDT against presumptive diagnosis was consistent: all the four studies that tested the robustness of the results found that RDT could be more cost-effective in the area with lower prevalence [30, 31, 39, 40].

\section{Age}

Among all included studies, eleven had no restriction on participants' age and four limits the population to students or children of different ages. Evidence showed that whether RDT could be cost-effective compared to other diagnostic methods was not likely to be influenced by the age of the target population. Of the four papers with a limitation on the age, half applied RDT on children under 5 years old and supported the cost-effectiveness of this diagnostic method [31, 40], while the other half focused on children as well and did not reach that conclusion, but both of them recognized the cost-saving effect of RDT compared to microscopy [28, 38]. Of eleven studies without a limitation on age, eight showed that RDT could be more cost-effective compared with other methods [27, 29, 30, 32, 35, 37, 39, 41]. The majority of economic evaluations included considered RDT as a cost-effective strategy regardless of whether the study limited the subjects' age. Further details can be seen in Additional files 2 and 3.

\section{The types of RDT}

There are various types of RDT: some of them can detect single Plasmodium species, some can detect multiple species and some can distinguish between different species [42]. The difference in the types may bring extra costs to the economic value of RDT as they may have different prices. To compare the impact of RDT types, we categorized RDT into two categories: one is a single test which only detects single species, another is a combo test which can detect multiple Plasmodium species. The types of RDT used in included studies varied greatly. Ten studies adopted single test $[27,28,30-35$, $39,40]$, while combo tests were used in seven studies $[28,29,32,36-38,41]$.

Evidence suggested that single RDT could be costeffective compared to microscopy and presumptive diagnosis. Plasmodium falciparum-specific RDTs were adopted in eight studies: four of them were decision analytical economic evaluations and suggested that the introduction of single RDT tests can largely improve the proportion of appropriate treatment for patients [27, 30, $31,40]$. In the other four studies, two of them found that 
RDT was likely to be more cost-effective than microscopy [35, 39], and the remaining two studies adopted single and multiple tests at the same time. In the first study conducted in Ethiopia where $P$. falciparum and $P$. vivax co-exist, both single and multiple tests were used to appraise the cost-effectiveness of RDT compared to presumptive treatment [32]. In the area with various malaria species, multiple tests were more cost-effective than either a single test or presumptive diagnosis. In a second study, a cost analysis was performed to appraise the performance of four RDT brands, including single and multiple tests, but it did not assess the effectiveness of multiple tests and only reported costs of general RDTs rather than costs by each RDT type [28].

However, the cost-effectiveness of combo tests was not clear. Four of seven studies showed positive results regarding the cost-effectiveness of combo RDT. Three studies that appraised the costs and effectiveness of RDT based on decision models observed lower costs and more clinical benefits with the use of multiple tests than microscopy $[29,37,41]$. Lemma et al. found that multiple tests performed better and cost lower than both single tests and presumptive diagnoses in the context where $P$. falciparum and $P$. vivax co-dominate [32]. However, the costeffectiveness of multiple tests applied in the remote area of Amazon where $P$. falciparum and $P$. vivax dominate as well were uncertain as it largely depended on the accessibility to and the accuracy of microscopy [36]. Evidence identified in this review observed that RDT could also lead to the problem of over-diagnosis [28,38]. Although RDT was the cheapest approach to detect infection in malaria school surveys compared to other strategies (i.e., microscopy or RDT corrected by alternative methods), it overestimated the prevalence of infection [28]. Also, the study only evaluated the costs of diagnosis and thus the costsaving effect of RDT could be maintained remainsed unclear when treatment costs were taken into account. The treatment costs were found to be higher for RDT than for microscopy when P. falciparum and pan-specific RDT was used to the management of malaria cases in Ghana [38]. The study also observed the over-diagnosis and additional costs when RDT was introduced to replace presumptive diagnosis. This may reduce RDT's advantage in terms of cost-effectiveness.

In general, the impact of the types of RDT on its costeffectiveness remained uncertain given various types of RDT, the complexity of local epidemiological characteristics and the lack of evidence reported in studies included. Further details of the types and brands of RDT can be seen in Table 1 .

\section{Funding sources}

Fourteen of all fifteen studies received funding from various sources (Additional file 4). It was not clear based on current evidence whether founding sources would have an impact on whether RDT was cost-effective. Seven studies were government-sponsored, either intergovernmental organization or local government [28, 32 , $34,35,37,39,41]$, and five of them supported the costeffectiveness of RDT [32, 35, 37, 39, 41]. Of eight studies that did not receive funding from the government [27, $29-31,33,36,38,40]$, seven were sponsored by either non-governmental organizations or research institutions including universities and five studies reported that RDT was cost-effective [27, 29-31, 40]. There was only one study that had no statement of the source of funding, and its result did not support RDT's cost-effectiveness because it found that if the accuracy of microscopy could be guaranteed, there would be no additional benefits of applying RDT [36]. As most of the studies included received funding from nonprofit organizations and there was only one research that did not report its funding source, the impact of funding sources was less clear.

\section{Discussion}

\section{Economic evidence for RDT}

Our study aimed at assessing the cost-effectiveness of RDT in a systematic manner. Overall, we identified fifteen studies that tried to delve out whether RDT was costeffective compared with other commonly used malaria diagnosis methods and there was heterogeneity in population age, funding sources, economic and effectiveness measures, and other general study settings across studies. Our analysis took the influence of such variability into account and found that most studies provided supportive evidence in terms of the cost-effectiveness of RDT.

However, there were still five studies that did not draw a clear conclusion [28, 33, 34, 36, 38]. This difference can be explained by the accuracy of RDT, the performance of its comparisons, clinicians' compliance with the diagnostic results, total treatment costs, and malaria prevalence. Therefore, we were unable to conclude which strategy would be the most cost-effective with certainty.

A wide range of perspectives has been selected by the studies included. Although most of the studies under the societal and health sector perspectives supported the cost-effectiveness of RDT, some could not because of the uncertainty in the costs and an unclear WTP threshold. This might suggest that the diagnosis and treatment of malaria can be unaffordable to patients in many countries. The recommended first-line malaria treatment, artemisinin-based combination therapy (ACT), is expensive and possible increase in treatment costs over time due to therapy resistance and drug prices has been seldom considered by researchers in the field trials. A costeffective intervention can be considered to receive public 
funding if it is a public good, or has important externalities and inadequate demand, or is catastrophically unaffordable and has no available insurance, or beneficiaries are poor when utility outcome is not available [43]. Given the expensive treatment costs, it is suggested that malaria case management with RDT should be included in the coverage of health insurance to substantially reduce the economic burden on patients and their families [44, 45].

Another key driver for the cost-effectiveness of RDT is its price $[27,30,31,33,36,41]$. The price of RDT can be determined by its type as combo RDTs are usually more costly than the single tests. In our analysis, it is uncertain whether the cost-effectiveness of RDT could be influenced by the types of RDT. Also, the capability of combo tests to identify plasmodium species can largely influence the cost-effectiveness of RDT because the type of RDT selected will determine not only the accuracy of diagnosis [46], but also the following treatment received. Therefore, for most countries where multiple malaria species dominate, it is necessary to differentiate Plasmodium species such that proper treatment could be delivered.

This systematic review included studies from low- and middle-income countries that were assumed to be malaria endemic. The cost-effectiveness of RDT compared to microscopy was not clear in regions with relatively low transmission settings given the uncertainty in how the routine microscopy was performed, i.e., the accuracy of microscopy and whether the microscope was used only for malaria detection. Current evidence suggested that RDT could be more cost-effective than microscopy $[29,37,39,41]$, and the relative advantage of RDT could be further enhanced if microscope was exclusively-used [37]. This could be explained by the fact that the demand for malaria diagnosis would be less in area where malaria prevalence is close to zero, and the cost per suspected patient would be largely increased when taking microscopy as the initial approach. Further studies are required to confirm this, especially in low transmission countries aiming at eliminating malaria.

Moreover, facing the reduction of malaria prevalence and movements towards disease elimination [47], it is more common for countries to confront the threat of increasing malaria imported cases [6]. Usually, imported patients are either rural migrant workers or travelers to the endemic region, and tend to have lower parasite densities. The key challenge is to promptly and accurately identify malaria cases at all levels of health systems. Current malaria control programs have established either active or passive case detection systems. Active case detection requires health workers to seek out for patients, making it less feasible to maintain the use of microscopy as the initial approach when the prevalence is extremely low. In addition to this, our requirements for malaria elimination, especially a consistent diagnostic accuracy for community-based primary care, is beyond the capacity of routine microscopy due to the scarcity of well-trained microscopists [48], and essential laboratory supplies. This may limit the performance of microscopy [49], and contribute to misdiagnosis or over-diagnosis with a potential risk of over-consuming antimalarial therapies and drug resistance [50]. In fact, the poor performance of routine microscopy has been widely recognized, even in developed countries [51], and high capital investment of microscopy makes it more costly than RDT if local caseload is low [52]. Therefore, it is meaningful and economically important to introduce RDT in primary health care or remote region where microscopy is unavailable.

\section{Quality of the evidence}

The CHEERS tool was used to assess the quality of evidence in our research, allowing to compare reporting quality across included studies. The majority of studies identified are of good and moderate quality, but we still found some studies showed poor compliance with the reporting guidance, especially lacking details of research methods. Explanation of model selection was lacked generally, and this might be because studies tended to have more concern about whether RDT was a quick and accurate way to detect malaria cases. It should also be noticed that the scores of studies aimed at measuring the costeffectiveness of RDT were higher than those only evaluating the costs of the disease detection approaches.

In addition, the CHEERS tool focuses on the quality of reporting, and it should be fully considered at the stage of study design, for example, by referring to the structural abstract proposed by NHS Economic Evaluation Database (NHS EED) and extracting basic characteristics and results of health economic evaluations to improve the quality of evidence.

\section{Limitations}

This review is limited in the following aspects: firstly, the studies identified were conducted in a limited range of countries, most of which were located in Africa. Whether results obtained from the context can be transferable to other countries was not elaborated in the included studies. It is obvious that countries may vary in their widely-used malaria diagnostic methods and other features such as prevalence and the types of RDT. Differences in health care systems and reimbursement also limit the transferability of our results. Thus, caution should be taken when applying the results to other settings. Another limitation was inadequate data on costs and effectiveness, possibly due to differences in adopting primary and secondary outcome indicators among studies, adding to the difficulty in comparing ICERs obtained 
when they had the same perspectives. Therefore, no synthesized outcome was shown due to the wide difference across studies and the lack of evidence regarding health utility gained when using different malaria diagnostic techniques. We suggest that further economic evaluations of malaria detection methods should focus on health utility benefits for patients who are susceptible to the disease.

\section{Conclusions}

We compared the cost-effectiveness of malaria RDT to other conventional diagnostic methods based on fifteen economic evaluations identified. However, there was high heterogeneity across economic evaluations identified in the outcome measures selection, the use of comparative diagnostic methods, and study settings. RDT was highly likely to be consistently cost-effective compared to presumptive diagnosis and routine microscopy, particularly in a low transmission setting. Further economic evaluations with better quality and comparable study designs were required.

\section{Supplementary information}

Supplementary information accompanies this paper at https://doi.org/10. 1186/s40249-019-0615-8.

Additional file 1. Methodological quality of all included studies.

Additional file 2. Economic results of the included studies: effectiveness measures, prevalence, original ICERs and adjusted ICERs (USD 2019).

Additional file 3. Economic results of the included studies: interventions, economic results and conclusions.

Additional file 4. Perspectives, funding sources and conflicts of interests of studies.

\section{Abbreviations}

ACT: Artemisinin-based combination therapy; CHEERS: Consolidated Health Economic Evaluation Reporting Standards; PCR: Polymerase chain reaction; RDT: Rapid diagnostic test; WHO: World Health Organization

\section{Acknowledgements}

Not applicable.

\section{Authors' contributions}

All authors outlined the scope of the review and were involved in the study design. $\mathrm{XL}$ and $J$ J designed the data extraction table, and independently reviewed studies identified, assessed the quality of studies and extracted data based on the table designed. $\mathrm{XL}$ wrote the first draft. JH made substantive suggestions for revision. All authors contributed to the final version of the manuscript.

\section{Funding}

This study supported by the Natural Science Foundation of Jiangsu Province (No. BK20150001), the Jiangsu Provincial Department of Science and Technology (BE2018020), and the Jiangsu Provincial Project of Invigorating Health Care through Science, Technology and Education. The funders had no role in the study design, data collection, analysis, decision to publish, or preparation of the manuscript.

\section{Availability of data and materials}

All data generated or analyzed in this study are included in this published article and its supplementary information files.
Ethics approval and consent to participate

Not applicable.

\section{Consent for publication}

Not applicable.

\section{Competing interests}

The authors declare that they have no competing interests.

\section{Author details}

${ }^{1}$ School of Public Health, Fudan University, Key Laboratory of Health Technology Assessment, National Health Commission, Shanghai 200032, China. ${ }^{2}$ National Health Commission Key Laboratory of Parasitic Disease Control and Prevention, Jiangsu Provincial Key Laboratory on Parasite and Vector Control Technology, Jiangsu Institute of Parasitic Diseases, Wuxi 214064, China. ${ }^{3}$ Center for Global Health, School of Public Health, Nanjing Medical University, Nanjing 211166, China. ${ }^{4}$ Public Health Research Centre, Jiangnan University, Wuxi 214122, China.

Received: 29 June 2019 Accepted: 9 December 2019

Published online: 30 December 2019

\section{References}

1. WHO. Disease surveillance for malaria elimination: Operational manual: World Health Organization; 2012. https://www.who.int/malaria/publications/ atoz/9789241503334/en/. Accessed 19 Aug 2019

2. WHO. Guidelines for the treatment of malaria. 3rd ed: World Health Organization; 2015. https://www.who.int/malaria/publications/atoz/ 9789241549127/en/. Accessed 19 Aug 2019

3. WHO. Malaria microscopy quality assurance manual - Ver. 2: World Health Organization; 2016. https://www.who.int/malaria/publications/atoz/ 9789241549394/en/. Accessed 19 Aug 2019

4. WHO. Global technical strategy for malaria 2016-2030: World Health Organization; 2015. https://www.who.int/malaria/publications/atoz/ 9789241564991/en/. Accessed 19 Aug 2019

5. Stäger K, Legros F, Krause G, Low N, Bradley D, Desai M, et al. Imported malaria in children in industrialized countries, 1992-2002. Emerg Infect Dis. 2009;15(2):185-91.

6. Cotter C, Sturrock H, Hsiang M, Liu J, Phillips A, Hwang J, et al. The changing epidemiology of malaria elimination: new strategies for new challenges. Lancet. 2013;382(9895):900-11.

7. Liu Y, Hsiang M, Zhou H, Wang W, Cao Y, Gosling R, et al. Malaria in overseas labourers returning to China: an analysis of imported malaria in Jiangsu Province, 2001-2011. Malar J. 2014;13(1):29.

8. Sabot O, Cohen J, Hsiang M, Kahn J, Basu S, Tang L, et al. Costs and financial feasibility of malaria elimination. Lancet. 2010;376(9752):1604-15.

9. Britton S, Cheng Q, McCarthy J. Novel molecular diagnostic tools for malaria elimination: a review of options from the point of view of high-throughput and applicability in resource limited settings. Malar J. 2016;15(1):88.

10. Tangpukdee N, Duangdee C, Wilairatana P, Krudsood S. Malaria Diagnosis: A Brief Review. Korean J Parasitol. 2009;47(2):93.

11. Murphy S, Prescott W, Stewart V, Parikh S, Etter P, Shott J. Malaria diagnostics in clinical trials. Am J Trop Med Hyg. 2013;89(5):824-39.

12. Ding G, Zhu G, Cao C, Miao P, Cao Y, Wang W, et al. The challenge of maintaining microscopist capacity at basic levels for malaria elimination in Jiangsu Province, China. BMC Public Health. 2018;18(1):489.

13. Murray C, Bell D, Gasser R, Wongsrichanalai C. Rapid diagnostic testing for malaria. Trop Med Int Health. 2003:8:876-83.

14. Doctor S, Liu Y, Whitesell A, Thwai K, Taylor S, Janko M, et al. Corrigendum to "Malaria surveillance in the Democratic Republic of the Congo: Comparison of microscopy, PCR, and rapid diagnostic test" [Diagn Microbiol Infect Dis. 2016 May;85(1):16-8.]. Diagn Microbiol Infect Dis. 2017;89(3):251.

15. Johnston S, Pieniazek N, Xayavong M, Slemenda S, Wilkins P, da Silva A. PCR as a confirmatory technique for laboratory diagnosis of malaria. J Clin Microbiol. 2006:44(3):1087-9.

16. Hänscheid T, Grobusch M. How useful is PCR in the diagnosis of malaria? Trends Parasitol. 2002;18(9):395-8.

17. Abba K, Deeks JJ, Olliaro PL, Naing CM, Jackson SM, Takwoingi Y, et al. Rapid diagnostic tests for diagnosing uncomplicated $P$. falciparum malaria in endemic countries. Cochrane Database Syst Rev. 2011:(7):CD008122. https://doi.org/10.1002/14651858.CD008122.pub2. 
18. Shretta R, Avanceña A, Hatefi A. The economics of malaria control and elimination: a systematic review. Malar J. 2016;15(1):593.

19. Higgins JPT, Green S. Cochrane handbook for systematic reviews of interventions version 5.1.0 (updated Mar 2011). Cochrane Collaboration. 2011; http://training.cochrane.org/handbook. Accessed 14 Mar 2017.

20. Laurent A, Schellenberg J, Shirima K, Ketende S, Alonso P, Mshinda H, et al. Performance of HRP-2 based rapid diagnostic test for malaria and its variation with age in an area of intense malaria transmission in southern Tanzania. Malar J. 2010;9(1):294

21. Bell C, Urbach D, Ray J, Bayoumi A, Rosen A, Greenberg D, et al. Bias in published cost effectiveness studies: systematic review. BMJ. 2006;332(7543): 699-703.

22. Abeku T, Kristan M, Jones C, Beard J, Mueller D, Okia M, et al. Determinants of the accuracy of rapid diagnostic tests in malaria case management: evidence from low and moderate transmission settings in the east African highlands. Malar J. 2008;7(1):202.

23. Eppi.ioe.ac.uk. CCEMG - EPPI-Centre Cost Converter v.1.6. https://eppi.ioe.ac. uk/costconversion/. Accessed15 Sept 2019.

24. Centre for Reviews and Dissemination. CRD's guidance for undertaking reviews in healthcare: CRD, University of York; 2009. https://www.york.ac.uk/ media/crd/Systematic_Reviews.pdf. Accessed 22 Apr 2019

25. Byford S, Raftery J. Perspectives in economic evaluation. BMJ. 1998; 316(7143):1529-30

26. Whitten PS, Mair FS, Haycox A, May CR, Williams TL, Hellmich S. Systematic review of cost effectiveness studies of telemedicine interventions. BMJ. 2002;324(7351):1434-7.

27. Batwala V, Magnussen P, Hansen K, Nuwaha F. Cost-effectiveness of malaria microscopy and rapid diagnostic tests versus presumptive diagnosis: implications for malaria control in Uganda. Malar J. 2011;10(1):372.

28. Gitonga CW, Kihara JH, Njenga SM, Awuondo K, Noor AM, Snow RW, et al. Use of rapid diagnostic tests in malaria school surveys in Kenya: does their under-performance matter for planning malaria control? Am J Trop Med Hyg. 2012;87:1004-11. https://doi.org/10.4269/ajtmh.2012.12-0215.

29. Hansen K, Grieve E, Mikhail A, Mayan I, Mohammed N, Anwar M, et al. Costeffectiveness of malaria diagnosis using rapid diagnostic tests compared to microscopy or clinical symptoms alone in Afghanistan. Malar J. 2015;14(1):217.

30. Hansen K, Clarke S, Lal S, Magnussen P, Mbonye A. Cost-effectiveness analysis of introducing malaria diagnostic testing in drug shops: a clusterrandomised trial in Uganda. PLoS One. 2017;12(12):e0189758.

31. Hansen K, Ndyomugyenyi R, Magnussen P, Lal S, Clarke S. Cost-effectiveness analysis of malaria rapid diagnostic tests for appropriate treatment of malaria at the community level in Uganda. Health Policy Plan. 2017;32(5): 676-89.

32. Lemma H, San Sebastian M, Löfgren C, Barnabas G. Cost-effectiveness of three malaria treatment strategies in rural Tigray, Ethiopia where both Plasmodium falciparum and Plasmodium vivax co-dominate. Cost Eff Resour Alloc. 2011;9(1):2.

33. Lubell $Y$, Reyburn $H$, Mbakilwa H, Mwangi R, Chonya K, Whitty MJC, Mills A. The cost-effectiveness of parasitologic diagnosis for malaria-suspected patients in an era of combination therapy. Am J Trop Med Hyg. 2007;77: 128-32

34. Ly A, Tall A, Perry R, Baril L, Badiane A, Faye J, et al. Use of HRP-2-based rapid diagnostic test for Plasmodium falciparum malaria: assessing accuracy and cost-effectiveness in the villages of Dielmo and Ndiop, Senegal. Malar J. 2010;9(1):153.

35. Matangila J, Lufuluabo J, Ibalanky A, Inocêncio da Luz R, Lutumba P, Van Geertruyden J. Asymptomatic Plasmodium falciparum infection is associated with anaemia in pregnancy and can be more cost-effectively detected by rapid diagnostic test than by microscopy in Kinshasa, Democratic Republic of the Congo. Malar J. 2014;13(1):132.

36. Oliveira MRF, Gomes AC, Toscano CM. Cost effectiveness of OptiMal ${ }^{\circledR}$ rapid diagnostic test for malaria in remote areas of the Amazon region, Brazil. Malar J. 2010;9:277

37. Oliveira MRF, Giozza SP, Peixoto HM, Romero GAS. Cost-effectiveness of diagnostic for malaria in extra-Amazon region, Brazil. Malar J. 2012;11:390.

38. Osei-Kwakye K, Asante KP, Mahama E, Apanga S, Owusu R, Kwara E, et al. The benefits or otherwise of managing malaria cases with or without laboratory diagnosis: the experience in a district hospital in Ghana. PLoS One. 2013;8(3):e58107. https://doi.org/10.1371/journal.pone.0058107.

39. Shillcutt S, Morel C, Goodman C, Coleman P, Bell D, Whitty CJM, et al. Costeffectiveness of malaria diagnostic methods in sub-Saharan Africa in an era of combination therapy. Bull World Health Organ. 2008;86:101-10. https:// doi.org/10.2471/BLT.07.042259.

40. Tawiah T, Hansen KS, Baiden F, Bruce J, Tivura M, Delimini R, et al. Costeffectiveness analysis of test-based versus presumptive treatment of uncomplicated malaria in children under five years in an area of high transmission in Central Ghana. PLoS One. 2016;11:e0164055. https://doi.org/ 10.1371/journal.pone.0164055.

41. Uzochukwu B, Obikeze E, Onwujekwe O, Onoka C, Griffiths U. Costeffectiveness analysis of rapid diagnostic test, microscopy and syndromic approach in the diagnosis of malaria in Nigeria: implications for scaling-up deployment of ACT. Malar J. 2009;8(1):265.

42. WHO. Rapid diagnostic tests. https://www.who.int/malaria/areas/diagnosis/ rapid diagnostic tests/en/ (2019). Accessed 28 Apr 2019.

43. Creese A, Floyd K, Alban A, Guinness L. Cost-effectiveness of HIV/AIDS interventions in Africa: a systematic review of the evidence. Lancet. 2002; 359(9318):1635-42.

44. Uzochukwu B, Onwujekwe O, Uguru N, Ughasoro M, Ezeoke O. Willingness to pay for rapid diagnostic tests for the diagnosis and treatment of malaria in Southeast Nigeria: ex post and ex ante. Int J Equity Health. 2010;9(1):1.

45. Tawiah T, Malam K, Kwarteng A, Bart-Plange C, Febir L, Aubyn V, et al. Improving the first-line treatment of febrile illnesses in Ghana: willingness to pay for malaria rapid diagnostic tests at licensed chemical shops in the Kintampo area. Cost Eff Resour Alloc. 2018;16(1):4.

46. Abba K, Kirkham AJ, Olliaro PL, Deeks JJ, Donegan S, Garner P, et al. Rapid diagnostic tests for diagnosing uncomplicated non-falciparum or Plasmodium vivax malaria in endemic countries. Cochrane Database Syst Rev. 2014;(12):CD011431. https://doi.org/10.1002/14651858.CD011431

47. WHO, FIND, CDC. Results of WHO product testing of malaria RDTs: Round 7 (2015-2016): World Health Organization; 2017. https://www. who.int/malaria/areas/diagnosis/rapid-diagnostic-tests/product-testinground7/en/. Accessed 28 Apr 2019

48. Aiyenigba B, Ojo A, Aisiri A, Uzim J, Adeusi O, Mwenesi H. Immediate assessment of performance of medical laboratory scientists following a 10day malaria microscopy training programme in Nigeria. Glob Health Res Policy. 2017;2(1):32

49. WHO. Technical consultation to update the WHO malaria microscopy quality assurance manual. Meeting report: World Health Organization; 2014. https://www.who.int/malaria/publications/atoz/microscopy-qualityassurance-report/en/. Accessed 28 Apr 2019

50. Odhiambo F, Buff A, Moranga C, Moseti C, Wesongah J, Lowther S, et al. Factors associated with malaria microscopy diagnostic performance following a pilot quality-assurance programme in health facilities in malaria low-transmission areas of Kenya, 2014. Malar J. 2017;16(1):371.

51. Wongsrichanalai C, Barcus MJ, Muth S, Sutamihardja A, Wernsdorfer WH. A review of malaria diagnostic tools: microscopy and rapid diagnostic test (RDT). Am J Trop Med Hyg. 2007;77:119-27. https://doi.org/10.4269/ajtmh. 2007.77.119.

52. Bell D, Wongsrichanalai C, Barnwell J. Ensuring quality and access for malaria diagnosis: how can it be achieved? Nat Rev Microbiol. 2006;4(9): 682-95.

Ready to submit your research? Choose BMC and benefit from

- fast, convenient online submission

- thorough peer review by experienced researchers in your field

- rapid publication on acceptance

- support for research data, including large and complex data types

- gold Open Access which fosters wider collaboration and increased citations

- maximum visibility for your research: over $100 \mathrm{M}$ website views per year

At $\mathrm{BMC}$, research is always in progress.

Learn more biomedcentral.com/submission 